\title{
CONFERENACIA CLINIICA Tratando os sintomas comportamentais e psicológicos da demência (SCPD)
}

\author{
Treating the behavioral and psychological symptoms of dementia (BPSD) \\ Paulo Caramelli', Cássio M. C. Bottino²
}

\section{RESUMO}

Os transtornos neuropsiquiátricos na demência, também denominados de sintomas comportamentais e psicológicos da demência (SCPD), têm prevalência elevada no curso clínico da doença de Alzheimer e de outras desordens relacionadas. A relevância dessas manifestações é bem reconhecida, estando relacionada a evolução clínica mais desfavorável, maior sobrecarga do cuidador e maior incidência de institucionalização, entre outros fatores. O objetivo desta conferência clínica é procurar responder a algumas questões relacionadas a este tema, com o intuito de oferecer aos leitores uma breve atualização sobre o assunto. Os seguintes tópicos foram selecionados para discussão: se a nomenclatura SCPD é adequada para denominar essas manifestações clínicas; se há uma forma de classificar e como diagnosticar esses sintomas; qual a sua prevalência, seu impacto no curso clínico das demências e quais os fatores de risco associados ao seu aparecimento; quais as bases fisiopatológicas conhecidas; e, finalmente, como abordar esses pacientes e também suas famílias e cuidadores, do ponto de vista terapêutico, tanto em relação a intervenções farmacológicas quanto não farmacológicas.

Palavras-chaves: Demência, sintomas psicológicos e comportamentais da demência, tratamento.

\begin{abstract}
Neuropsychiatric disturbances in dementia, also named behavioral and psychological symptoms of dementia (BPSD), are highly prevalent in the clinical course of Alzheimer disease and related disorders. The clinical relevance of these manifestations is well recognized, being related to a poor clinical outcome, greater caregiver burden and to higher institutionalization rates, among others. This clinical conference attempts to respond to some questions related to this subject, in order to offer a brief update to the readers. The following topics are discussed: if the nomenclature BPSD is adequate for these clinical manifestations; how to classify and to diagnose these symptoms; how prevalent they are; which is their impact on the clinical course of the dementias and which are the risk factors associated to their emergence; which are their currently known pathophysiological basis; and, finally, which are the available pharmacological and non-pharmacological interventions, both for the patients and for their families and caregivers.
\end{abstract}

Key-words: Dementia, behavioral and psychological symptoms of dementia, treatment.

\section{INTRODUÇÃO}

U ma boa forma de começarmos esta conferência seria questionando se a denominação sintomas comportamentais e psicológicos da demência, uma tradução direta do inglês behavioral and psychological symptoms of dementia (BPSD), poderia ser considerada uma expressão adequada para definir as manifestações neuropsiquiátricas dos transtornos demenciais. A denominação originalmente proposta por Finkel et al. (1996), a partir de uma reunião de consenso de especialistas em Neuropsiquiatria Geriátrica realizada na Austrália, em 1995, foi sintomas e sinais comportamentais e psicológicos da demência. Essa terminologia refere-se ao conjunto de sintomas e sinais relacionados a transtornos da percepção, do conteúdo do pensamento, do humor ou do comportamento que ocorrem freqüentemente em pacientes com síndrome demencial (Finkel et al., 1996).

A introdução dessa nomenclatura na literatura científica teve o inquestionável mérito de chamar a atenção de pesquisadores e clínicos para a relevância de tais manifestações como parte integrante da síndrome demencial, uma vez que estas eram menos valorizadas do que as alterações cognitivas. No entanto, pouco tempo após a publicação desse consenso, o termo "sinais" acabou sendo esquecido, o que restringiu a abrangência do conceito inicialmente proposto. Provavelmente isso se deu apenas pela maior facilidade no emprego do termo/sigla e não por qualquer motivo científico, mas passados 11 anos da publicação do consenso, a denominação original acabou sendo modificada, o que gera uma incorreção. Pacientes com demência apresentam comprometimento de memória e de outras funções cognitivas, o que muitas vezes os impede, especialmente em fases mais avançadas, de efetuar um relato adequado de seu estado emocional interno (sintomas) e faz que comportamentos observados por familiares ou cui-

$\begin{array}{ll} & 1 \text { Faculdade de Medicina da Universidade Federal de Minas Gerais (UFMG). } \\ & \text { 2 Hospital das Clínicas da Faculdade de Medicina da Universidade de São Paulo (FMUSP). } \\ \text { Recebido } & \\ \text { Ap/02/2007 } & \\ \text { 18/06/2007 } & \\ & \text { Endereço para correspondência: Paulo Caramelli } \\ \text { Av. Prof. Alfredo Balena, 190, Sala 246-30130-100 - Belo Horizonte, MG } & \text { Telefax: (31) 3248-9746 } \\ \text { E-mail: caramelp@usp.br }\end{array}$


dadores (sinais) sejam mais facilmente referidos (McKeith e Cummings, 2005).

Outra limitação do termo proposto é que, ao listarem os tipos de alterações de comportamento apresentados pelos pacientes, os autores não citaram a apatia, que, segundo diversos estudos, é a manifestação neuropsiquiátrica mais comum nas demências, especialmente na doença de Alzheimer (DA) (Lyketsos et al., 2002; Tatsch et al., 2006). Por outro lado, alguns sintomas mencionados podem ser considerados síndromes. Um exemplo é a agitação, que inclui ansiedade, irritabilidade, inquietação motora e vocalização anormal. Ou a própria apatia, em que ocorrem embotamento afetivo, perda de interesse e de iniciativa, isolamento social e disfunção executiva (Teixeira Jr. e Caramelli, 2006).

A despeito de a sigla BPSD (ou SCPD) ter se tornado bastante popular e inegavelmente útil do ponto de vista científico e clínico, é possível que o emprego de uma terminologia mais abrangente, como "manifestações neuropsiquiátricas da demência", possa minimizar eventuais desvios de interpretação.

O objetivo dessa conferência clínica é procurar responder a algumas questões relacionadas a esse tema com o intuito de oferecer aos leitores uma breve atualização sobre o assunto.

\section{Classificação e apresentação clínica dos sintomas}

Nos últimos anos, pesquisadores da área têm proposto a utilização de classificações mais específicas, para facilitar a realização de estudos e a avaliação de intervenções terapêuticas. Por exemplo, Jeste e Finkel (2000) propuseram critérios específicos para o diagnóstico da psicose da DA e desordens relacionadas e Olin et al. (2002) descreveram um critério diagnóstico provisório para depressão na DA. Em 198 pacientes com DA, diagnosticados no estudo epidemiológico realizado em Cache County, nos EUA, Lyketsos et al. (2001) descreveram que, por meio da análise de classes latentes, o espectro das manifestações neuropsiquiátricas nos pacientes com DA poderia ser empiricamente dividido em três grupos: síndrome afetiva, síndrome psicótica e outros distúrbios neuropsiquiátricos. Entretanto, como bem destacaram esses autores, a validade preditiva e a biológica dessas classificações devem ser estudadas com maior profundidade (Lyketsos et al., 2001).

\section{Avaliação clínica dos pacientes}

O emprego de instrumentos de avaliação cognitiva, especialmente testes de rastreio ou de avaliação neuropsicológica breve, é atualmente bastante difundido na prática clínica. No entanto, a avaliação quantitativa das manifestações neuropsiquiátricas em pacientes com demência é ainda bastante restrita. Além da identificação dos diferentes tipos de alteração, é importante determinar sua intensidade e freqüência, bem como o desgaste ou a sobrecarga que representam para o familiar ou o cuidador.

Há escalas de avaliação especificamente elaboradas para pacientes com demência. Entre estas, podemos destacar a Escala de Avaliação de Comportamentos Patológicos na DA (BEHAVE-AD; Reisberg et al., 1987) e o Inventário Neuropsiquiátrico (NPI; Cummings et al., 1994). Tanto a BEHAVE-AD quanto o NPI são administrados ao familiar/cuidador, sob forma de entrevistas semi-estruturadas.

A BEHAVE-AD pontua 25 sintomas agrupados em sete domínios neuropsiquiátricos: 1. ideação paranóide e delirante; 2. alucinações; 3 . distúrbios da atividade; 4. agressividade; 5. distúrbios do ritmo circadiano; 6 . distúrbios da afetividade; 7. ansiedades e fobias. As informações são referentes as 2 semanas que antecedem a avaliação e para cada sintoma há quatro alternativas (de 0 a 3, em ordem crescente de intensidade). $O$ escore total na escala varia de 0 a 75 pontos.

O NPI, por sua vez, avalia 12 domínios distintos: 1. delírios; 2. alucinações; 3. agitação; 4. depressão/disforia; 5. ansiedade; 6. euforia/elação; 7. apatia/indiferença; 8. desinibição; 9. irritabilidade/labilidade; 10. comportamento motor aberrante; 11. comportamentos noturnos; 12. apetite/alterações alimentares. Cada item é avaliado em relação à sua freqüência ( 1 = ausente a 4 = muito freqüente) e intensidade $(1=$ leve a 3 = acentuada). 0 escore total é obtido pela multiplicação da freqüência pela intensidade, podendo variar de 0 a 144 pontos. O desgaste ou sobrecarga do cuidador também é avaliado, mas não é computado no escore total.

Embora esses dois instrumentos diagnósticos sejam essencialmente utilizados em protocolos de pesquisa, também podem - dependendo da disponibilidade de tempo durante a consulta - ser empregados na prática clínica, permitindo, por exemplo, medir de forma objetiva e quantitativa o impacto de uma determinada intervenção terapêutica.

\section{Epidemiologia dos SCPD}

A prevalência dos SCPD pode variar bastante, dependendo do tipo de amostra estudada e dos métodos utilizados para avaliá-los. Entretanto, em alguns estudos comunitários, avaliou-se a prevalência dos SCPD sistematicamente, como em outra publicação do mesmo estudo citado anteriormente (Lyketsos et al., 2002), no qual foram encontrados sintomas neuropsiquiátricos em $75 \%$ dos pacientes com demência, sendo as alterações mais comuns apatia (36\%), depressão (32\%) e agitação/agressividade (30\%). Hwang et al. (2004) avaliaram os SCPD em 124 pacientes com DA leve, relatando que $89 \%$ dos pacientes apresentavam ao menos um sintoma neuropsiquiátrico, sendo os sintomas mais freqüentes apatia (51\%), disforia (50\%) e irritabilidade (38\%). Geda et al. (2004) avaliaram 87 pacientes com DA leve, encontrando em $80 \%$ deles ao menos um sintoma neuropsiquiátrico, entre os quais os mais freqüentes foram apatia (37,9\%), irritabilidade $(35,6 \%)$ e depressão $(32,2 \%)$. 
Em relação à investigação da freqüência dos SPCD em estudos epidemiológicos brasileiros, podemos citar alguns dados que obtivemos em estudo realizado na cidade de São Paulo, para investigar a prevalência de demência em uma amostra comunitária $(n=1.563)$, com mais de 60 anos de idade. A taxa observada de prevalência de demência foi de $6,8 \%$, e $59,8 \%$ tinham DA (Bottino et al., 2005). Nos 60 pacientes com DA avaliados, os informantes relataram que $78,3 \%$ apresentavam um ou mais sintomas neuropsiquiátricos, entre os quais apatia (53,3\%), depressão (38,3\%), alterações do sono (38,3\%), ansiedade (25\%) e agitação/ agressividade (20\%) foram os mais prevalentes. Portanto, esses dados sugerem que os sintomas neuropsiquiátricos parecem ser comuns e com um perfil bastante semelhante tanto em pacientes que residem em países desenvolvidos quanto naqueles com DA vivendo em países em desenvolvimento como o Brasil (Tatsch et al., 2006).

\section{O impacto dos SCPD na evolução clínica das demências}

A maioria dos estudos a esse respeito realizou-se com pacientes com DA, demonstrando que a ocorrência de manifestações psicóticas se correlaciona com curso clínico mais desfavorável. Em uma revisão de 55 estudos publicados entre 1990 e 2003 sobre psicose na DA, os autores relatam que em todos os estudos identificados em que essa relação foi investigada ( $n=9$ ) se verificou uma associação direta e significativa entre a presença de manifestações psicóticas e evolução mais rápida da doença (Ropacki e Jeste, 2005). A análise do impacto de delírios e alucinações separadamente sugere que apenas estas últimas se associam a pior prognóstico (Burns et al., 1990a, 1990b; Wilson et al., 2000).

Da mesma forma, recentemente se descreveu a associação significativa entre a presença de apatia e declínio mais rápido tanto do ponto de vista cognitivo quanto funcional em pacientes com DA (Starkstein et al., 2006), desfechos estes que também parecem correlacionar-se com a presença de sintomas depressivos no curso da doença (Rapp et al., 2006).

Outros aspectos relevantes quanto ao impacto dos SCPD são a sua associação com maiores taxas de institucionalização (Yaffe et al., 2002), maior sobrecarga dos cuidadores (Rinaldi et al., 2005), especialmente na demência frontotemporal (Vugt et al., 2006), e menor sobrevida de pacientes com DA (Weiner et al., 2005).

\section{Fatores de risco associados ao aparecimento de SCPD}

Em estudo recente (Steinberg et al., 2006), no qual se reavaliou uma parte da amostra investigada no estudo de Cache County ( $n=184)$ após 18 meses, as mulheres mostraram tendência aumentada de apresentar ansiedade (razão de chances - RC: 2,22) e delírios (RC: 2,15$)$, mas as pessoas idosas de ambos os sexos demonstraram menor tendência de apresentar ansiedade.
Com o incremento da gravidade de demência, aumentou a tendência de apresentar alucinações e agitação $(R C: 2,42)$ e diminuiu o risco de depressão. A presença do alelo APOE $\varepsilon 4$ elevou a tendência de comportamento motor aberrante (RC: 1,84), e entre os diagnósticos de demência, os pacientes com DA mostraram menor tendência a apresentar agitação (RC: 0,58), depressão (RC: 0,56) e desinibição (RC: 0,46).

Maior tempo de observação elevou o risco da ocorrência de comportamento motor aberrante e delírios, e comorbidades médicas mais graves aumentaram o risco da ocorrência de agitação, irritabilidade, desinibição e comportamento motor aberrante. Portanto, nessa amostra representativa de uma população de idosos com demência, o gênero, a idade, a gravidade da demência, o alelo APOE $\varepsilon 4$, o tipo de demência, o tempo de seguimento e as comorbidades médicas influenciaram a ocorrência de determinados sintomas neuropsiquiátricos (Steinberg et al., 2006). Outros estudos de seguimento são necessários para identificar fatores de risco para os SCPD que sejam potencialmente modificáveis.

\section{Fisiopatologia e áreas cerebrais envolvidas no aparecimento dos sintomas}

$\mathrm{Na}$ DA, três manifestações neuropsiquiátricas já foram objeto de estudo quanto à identificação de suas bases neurobiológicas: depressão, apatia e delírios. Pacientes com DA e sintomas depressivos apresentaram redução significativa do metabolismo, medido por meio de tomografia por emissão de pósitrons (PET), no córtex frontal superior direito (Lee et al., 2006), e maiores densidades de placas senis e de emaranhados neurofibrilares (lesões neuropatológicas características da doença) na formação hipocampal (Rapp et al., 2006), em comparação com casos de DA sem depressão. A presença de apatia, por sua vez, se correlaciona com hipofluxo, à tomografia por emissão de fóton único (SPECT), no cíngulo anterior (Migneco et al., 2001; Robert et al., 2006). Essa relação anatômica é corroborada pela observação de associação entre o acúmulo de emaranhados neurofibrilares no cíngulo anterior e maior gravidade de apatia em pacientes com DA submetidos à autópsia (Tekin et al., 2001).

Nesse mesmo estudo, observaram-se correlação entre agitação e comportamento motor aberrante e maior intensidade de patologia neurofibrilar no córtex orbitofrontal.

Delírios na DA parecem estar relacionados à menor atividade em áreas corticais pré-frontais, especialmente à direita, achado este observado em dois estudos independentes, um utilizando SPECT e outro, PET (Sultzer et al., 2003; Nakano et al., 2006).

Também se descreve a associação entre maior comprometimento de áreas frontais (e temporais anteriores) direitas e maior intensidade de manifestações neuropsiquiátricas na demência frontotemporal, particularmente na sua variante frontal (Liu et al., 2004). 
Na demência com corpos de Lewy, em que é comum a ocorrência de alucinações visuais, é freqüente a observação de hipofluxo occipital ao SPECT, indicando acometimento de áreas corticais primárias e secundárias relacionadas ao processamento visual (Minoshima et al., 2001). Adicionalmente, pacientes com essa forma de demência que apresentam alucinações no curso da doença exibem, à autópsia, maior densidade de corpos de Lewy em lobo temporal e amígdala, quando comparados a pacientes sem histórico de alucinações (Harding et al., 2002).

\section{Abordagens terapêuticas gerais e psicológicas dos SCPD}

O primeiro passo na abordagem dos SCPD é identificar e quantificar as alterações neuropsiquiátricas e avaliar a possibilidade de serem secundárias a comorbidades que são especialmente freqüentes em idosos com demência, como infecção (sobretudo do trato urinário ou respiratório, muitas vezes sem febre), desidratação, descompensação metabólica, efeitos adversos de drogas, fraturas, traumatismo craniano, entre outras.

Uma vez afastadas tais possibilidades, a intervenção terapêutica deve ser sempre iniciada por medidas não farmacológicas, que incluem adaptações ou modificações ambientais, instituição de rotinas específicas para os pacientes, orientações dirigidas aos cuidadores e familiares, além de programas de atividade física leve (por ex., caminhadas), emprego de música e terapia de luz. Muitos comportamentos inapropriados respondem positivamente a tais medidas e merecem consideração especial pelo clínico.

\section{Abordagens familiares para os SCPD}

O impacto da ocorrência dos SCPD sobre a família dos pacientes com demência é muito elevado e, portanto, devemos dar especial atenção aos familiares e cuidadores, que terão que enfrentar mais essa sobrecarga associada ao cuidado desses doentes. É fundamental orientar o familiar/cuidador que procure identificar se existe algum fator associado ao aparecimento do sintoma ou comportamento alterado. Eventualmente, pedimos ao familiar que registre o dia e o horário em que o comportamento ocorreu, se havia algum desencadeante, que tipo de comportamento ou sintoma se observou e as conseqüências desse comportamento (Omelan, 2006).

Dessa maneira, muitas vezes é possível identificar padrões que podem permitir mudanças no ambiente físico e no manejo das situações associadas aos sintomas, introduzindo estímulos ou distrações que podem reduzir os SCPD. Por exemplo, música suave durante o banho, higiene pessoal ou troca de roupas pode tranqüilizar o paciente e facilitar a realização dessas tarefas pelo cuidador/familiar. Outra orientação importante é a recomendação de que se tente evitar ao máximo o confronto com o paciente, procurando oferecer alternativas, outros estímulos ou atividades que possam interessá-lo e permitir que os cuidados necessários sejam adequadamente prestados pelo familiar/cuidador.
De qualquer maneira, o profissional deve estar sempre atento ao surgimento de sintomas e transtornos psiquiátricos no familiar, procurando encaminhá-lo ao atendimento psicoterápico de apoio ou suporte, ou até mesmo para tratamento farmacoterápico, sempre que julgar necessário.

\section{Recomendações quanto ao tratamento farmacológico para os SCPD}

A primeira recomendação no tratamento dos SCPD é a de instituir o manejo não farmacológico, tranqüilizando e educando os familiares/cuidadores, procurando os desencadeantes dos comportamentos alterados e promovendo mudanças no ambiente físico e na rotina para tentar reduzir os sintomas e/ou comportamentos alterados. Opie et al. (1999) revisaram 43 estudos, incluindo cinco ensaios clínicos randomizados (ECR), para investigar a eficácia das abordagens não farmacológicas no tratamento dos SCPD.

Os autores concluíram que, apesar de vários problemas metodológicos identificados, existiriam evidências de eficácia em programas com atividades, música, terapia comportamental, terapia com luz, educação do cuidador e mudanças no ambiente físico.

Entretanto, quando as abordagens não farmacológicas não são suficientes para reduzir ou controlar os sintomas, quando os SCPD são muito intensos ou ocasionam muito sofrimento ao paciente e a seus familiares/cuidadores, o clínico deverá escolher um fármaco, procurando sempre ter em mente algumas questões fundamentais que devem anteceder o início do tratamento: a) Qual é o problema de comportamento a ser tratado?; b) Esta é a menor dose possível do medicamento? c) Este fármaco possui efeitos adversos que podem ocorrer com maior probabilidade em idosos?; d) Esta é a escolha com a meIhor relação custo-benefício?; e) Por quais critérios e em quanto tempo os efeitos terapêuticos serão avaliados? (Omelan, 2006).

Em relação aos psicofármacos, os antipsicóticos, antidepressivos, anticonvulsivantes, estabilizadores do humor, inibidores das colinesterases e a memantina são as principais opções para o tratamento dos SCPD. Em uma revisão do tratamento farmacológico dos SCPD, Sink et al. (2005) destacaram em relação a cada uma dessas opções que: a) Antipsicóticos típicos (duas meta-análises e dois ECR): sem diferença entre agentes específicos, com eficácia pequena e efeitos adversos comuns; b) Antipsicóticos atípicos (seis ECR): eficácia modesta significativa de olanzapina e risperidona, com efeitos adversos mínimos em baixas doses; os antipsicóticos atípicos estariam associados a aumento do risco para acidente vascular cerebral; c) Antidepressivos (cinco ECR): eficácia apenas para os sintomas depressivos, com aparente exceção para o citalopram; d) Estabilizadores do humor: três ECR com valproato, sem eficácia, e dois ECR com carbamazepina, com resultados conflitantes; e) duas meta-análises e seis ECR com inibidores das colinesterases mostraram eficácia pequena, 
mas significativa; f) dois ECR com memantina mostraram resultados conflitantes no tratamento dos SCPD.

Portanto, esses resultados baseados em evidências científicas, combinados com a experiência clínica de cada profissional e a análise detalhada de cada paciente a ser tratado, podem servir de parâmetro para os clínicos no momento de prescrever os psicofármacos para tratar OS SCPD.

\section{CONCLUSÕES}

Podemos concluir que os SCPD são manifestações clínicas relevantes e que, quando presentes, podem conferir maior

\section{REFERÊNCIAS}

Bottino CMC et al. Prevalence of dementia and MCl in São Paulo, Brazil. Int Psychogeriatr, 17: 80, 2005.

Burns A, Jacoby R, Levy R. Psychiatric phenomena in Alzheimer's disease. I: disorders of thought content. Br J Psychiatry, 157:72-6, 1990a.

Burns A, Jacoby R, Levy R. Psychiatric phenomena in Alzheimer's disease. II: disorders of perception. Br J Psychiatry, 157:76-81, 1990b.

Cummings $\rfloor$ et al. The neuropsychiatric inventory: comprehensive assessment of psychopathology in dementia. Neurology, 44:2308-14, 1994.

Finkel SI et al. Behavioral and psychological signs and symptoms of dementia: a consensus statement on current knowledge and implications for research and treatment. Int Psychogeriatr, 8 (Suppl. 3):497-500, 1996.

Geda YE, Smith GE, Knopman DS. De novo genesis of neuropsychiatric symptoms in mild cognitive impairment (MCI). Int Psychogeriatr, 16:51-60, 2004.

Harding AJ et al. Clinical correlates of selective pathology in the amygdala of patients with Parkinson's disease. Brain, 125:2431-45, 2002.

Hwang TJ et al. Mild cognitive impairment is associated with characteristic neuropsychiatric symptoms. Alzheimer Dis Assoc Disord, 18:17-21, 2004.

Jeste D, Finkel S. Psychosis of Alzheimer's disease and related dementias diagnostic criteria for a distinct syndrome. Am J Geriatr Psychiatry, 8:29-34, 2000.

Lee DY et al. Frontal dysfunction underlies depressive syndrome in Alzheimer disease: a FDG-PET study. Am J Geriatr Psychiatry, 14:625-8, 2006.

Liu W et al. Behavioral disorders in the frontal and temporal variants of frontotemporal dementia. Neurology, 62:742-48, 2004.

Lyketsos CG et al. Neuropsychiatric disturbance in Alzheimer's disease clusters into three groups: the Cache County study. Int J Geriat Psychiatry, 16:1043-53, 2001.

Lyketsos C $G$ et al. Prevalence of neuropsychiatric symptoms in dementia and mild cognitive impairment: results from the cardiovascular health study. JAMA, 288:1475-83, 2002.

McKeith I, Cummings J. Behavioural changes and psychological symptoms in dementia disorders. Lancet Neurol, 4:735-42, 2005.

Migneco 0 et al. Perfusion brain SPECT and statistical parametric mapping analysis indicate that apathy is a cingulate syndrome: a study in Alzheimer's disease and nondemented patients. Neuroimage, 13:896-902, 2001.

Minoshima S et al. Alzheimer's disease versus dementia with Lewy bodies: cerebral metabolic distinction with autopsy confirmation. Ann Neurol, 50:358-65, 2001.

Nakano S et al. Relationship between delusions and regional cerebral blood flow in Alzheimer's disease. Dement Geriatr Cogn Disord, 21:16-21, 2006. gravidade ao quadro. O profissional de saúde deve estar atento a sua ocorrência e, quando diagnosticados os SCPD, devem ser acompanhados e tratados de maneira adequada. A abordagem apropriada dessas condições pode levar à melhor condução clínica do caso, diminuindo o sofrimento para o paciente e os cuidadores.

Potenciais conflitos de interesse: Paulo Caramelli recebeu anteriormente honorários das empresas farmacêuticas Apsen, Janssen-Cilag, Novartis e Wyeth, referentes a aulas ministradas, à produção de materiais de conteúdo científico para divulgação à classe médica e à participação em ensaios clínicos.
Olin JT et al. Provisional diagnostic criteria for depression in Alzheimer's disease. Am J Geriatr Psychiatry, 10:125-28, 2002.

Omelan C. Approach to managing behavioural disturbances in dementia. Can Fam Physician, 52:191-99, 2006.

Opie J, Rosewarne R, O'Connor DW. The efficacy of psychosocial approaches to behaviour disorders in dementia: a systematic literature review. Aust N Z J Psychiatry, 33:789-99, 1999.

Rapp MA et al. Increased hippocampal plaques and tangles in patients with Alzheimer disease with a lifetime history of major depression. Arch Gen Psychiatry, 63:161-7, 2006.

Reisberg B et al. Behavioral symptoms in Alzheimer's disease: phenomenology and treatment. J Clin Psychiatry, 48 (Suppl.):9-15, 1987.

Rinaldi P et al. Predictors of high level of burden and distress in caregivers of demented patients: results of an Italian multicenter study. Int J Geriatr Psychiatry, 20:168-74, 2005.

Robert PH et al. Lack of initiative and interest in Alzheimer's disease: a single photon emission computed tomography study. Eur J Neurol, 13:729-35, 2006

Ropacki SA, Jeste DV. Epidemiology of and risk factors for psychosis of Alzheimer's disease: a review of 55 studies published from 1990 to 2003. Am J Psychiatr, 162:2022-30, 2005.

Sink KM, Holden KF, Yaffe K. Pharmacological treatment of neuropsychiatric symptoms of dementia. A review of the evidence. JAMA, 293:596-608, 2005.

Starkstein SE et al. A prospective longitudinal study of apathy in Alzheimer's disease. J Neurol Neurosurg Psychiatry, 77:8-11, 2006.

Steinberg M et al. Risk factors for neuropsychiatric symptoms in dementia: the Cache County Study. Int J Geriatr Psychiatry, 21:824-30, 2006.

Sultzer DL et al. Delusional thoughts and regional frontal/temporal cortex metabolism in Alzheimer's disease. Am J Psychiatry, 160:341-9, 2003

Tatsch MF et al. Neuropsychiatric symptoms in Alzheimer disease and cognitively impaired, nondemented elderly from a community-based sample in Brazil: prevalence and relationship with dementia severity. Am J Geriatr Psychiatry, 14:438-45, 2006.

Teixeira-Jr AL, Caramelli P. Apatia na doença de Alzheimer. Rev Bras Psiquiatr, 28:238-41, 2006.

Tekin S et al. Orbitofrontal and anterior cingulate cortex neurofibrillary tangle burden is associated with agitation in Alzheimer's disease. Ann Neurol, 49:355-61, 2001.

Weiner MF et al. Early behavioral symptoms and course of Alzheimer's disease. Acta Psychiatr Scand, 111:367-71, 2005.

Wilson RS et al. Hallucinations, delusions, and cognitive decline in Alzheimer disease. J Neurol Neurosurg Psychiatry, 69:172-7, 2000.

Yaffe K et al. Patient and caregiver characteristics and nursing home placement in patients with dementia. JAMA, 287:2090-7, 2002.

VugtMEetal. Impact of behavioural problems on spousal caregivers: a comparison between Alzheimer's disease and frontotemporal dementia. Dement Geriatr Cogn Disord, 22:35-41, 2006. 